REVISTA DE LA

UNIÓN MATEMÁTICA ARGENTINA

Vol. 59, No. 1, 2018, Pages 179-192

Published online: November 6, 2017

\title{
METALLIC CONJUGATE CONNECTIONS
}

\author{
ADARA M. BLAGA AND CRISTINA-ELENA HREŢCANU
}

Dedicated to the memory of Professor Dr. Vera W. de Spinadel

\begin{abstract}
Properties of metallic conjugate connections are stated by pointing out their relation to product conjugate connections. We define the analogous in metallic geometry of the structural and the virtual tensors from the almost product geometry and express the metallic conjugate connections in terms of these tensors. From an applied point of view we consider invariant distributions with respect to the metallic structure and for a natural pair of complementary distributions, the above structural and virtual tensors are expressed in terms of O'Neill-Gray tensor fields.
\end{abstract}

\section{INTRODUCTION}

Besides the very well known almost complex, almost tangent, and almost product structures on a differentiable manifold $M$, some other polynomial structures naturally arise as $C^{\infty}$-tensor fields $J$ of type $(1,1)$ which are roots of the algebraic equation

$$
Q(J):=J^{n}+a_{n} J^{n-1}+\cdots+a_{2} J+a_{1} I_{\mathfrak{X}(M)}=0,
$$

where $I_{\mathfrak{X}(M)}$ is the identity map on the Lie algebra of vector fields on $M$. In particular, if the structure polynomial is $Q(J):=J^{2}-p J-q I_{\mathfrak{X}(M)}$, with $p$ and $q$ positive integers, its solution $J$ will be called metallic structure [11]. The name is motivated by the fact that, for different values of $p$ and $q$, the $(p, q)$-metallic number introduced by Vera W. de Spinadel [14] is precisely the positive root of the quadratic equation $x^{2}-p x-q=0$, namely $\sigma_{p, q}=\frac{p+\sqrt{p^{2}+4 q}}{2}$. For example:

- for $p=q=1$ we get the golden number $\sigma=\frac{1+\sqrt{5}}{2}$ which is the limit of the ratio of two consecutive Fibonacci numbers [13];

- for $p=2$ and $q=1$ we get the silver number $\sigma_{2,1}=1+\sqrt{2}$ which is the limit of the ratio of two consecutive Pell numbers [9];

- for $p=3$ and $q=1$ we get the bronze number $\sigma_{3,1}=\frac{3+\sqrt{13}}{2}$ which plays an important role in the study of dynamical systems and quasicrystals;

- for $p=1$ and $q=2$ we get the copper number $\sigma_{1,2}=2$;

2010 Mathematics Subject Classification. 53C07, 53C15, 53C25.

Key words and phrases. Metallic structure; (conjugate) linear connection; metallic Riemannian manifold; structural and virtual tensor field; invariant distributions. 
- for $p=1$ and $q=3$ we get the nickel number $\sigma_{1,3}=\frac{1+\sqrt{13}}{2}$, and so on.

Notice that if $J$ is a metallic structure on $M$ with $J^{2}=p J+q I_{\mathfrak{X}(M)}$, then $\tilde{J}:=$ $a J+b I_{\mathfrak{X}(M)}$ is also a metallic structure with $\tilde{J}^{2}=\tilde{p} \tilde{J}+\tilde{q} I_{\mathfrak{X}(M)}$, for $a=\frac{2 \sigma_{\tilde{p}, \tilde{q}}-\tilde{p}}{2 \sigma_{p, q}-p}$ and $b=\frac{\tilde{p}}{2}-\frac{p}{2} \cdot \frac{2 \sigma_{\tilde{p}, \tilde{q}}-\tilde{p}}{2 \sigma_{p, q}-p}$.

In this setting, we shall study the properties of the conjugate connections (by a metallic structure), express their virtual and structural tensor fields and study their behavior on invariant distributions. Finally, taking into account the connection between metallic and almost product structures stated in [12, we shall analyze the impact of the duality between the metallic and almost product structures on metallic and product conjugate connections.

Let $M$ be a smooth, $n$-dimensional manifold and denote by $C^{\infty}(M)$ the algebra of smooth real functions on $M$, by $\mathfrak{X}(M)$ the Lie algebra of vector fields on $M$, and by $T_{s}^{r}(M)$ the $C^{\infty}(M)$-module of tensor fields of $(r, s)$-type on $M$. Usually $X, Y, Z, \ldots$ will be vector fields on $M$ and if $T \rightarrow M$ is a vector bundle over $M$, then $\Gamma(T)$ denotes the $C^{\infty}$-module of sections of $T$, e.g. $\Gamma(T M)=\mathfrak{X}(M)$.

Consider $\mathcal{C}(M)$ the set of all linear connections on $M$. Since the difference of two linear connections is a tensor field of $(1,2)$-type, it results that $\mathcal{C}(M)$ is a $C^{\infty}(M)$-affine module associated to the $C^{\infty}(M)$-linear module $T_{2}^{1}(M)$.

Recall the concept of metallic (Riemannian) geometry:

Definition $1.1([12]) . J \in T_{1}^{1}(M)$ is called metallic structure on $M$ if it satisfies the equation

$$
J^{2}=p J+q I_{\mathfrak{X}(M)},
$$

for $p, q \in \mathbb{N}^{*}$, where $I_{\mathfrak{X}(M)}$ is the identity operator on $\mathfrak{X}(M)$. The pair $(M, J)$ is a metallic manifold. Moreover, if a Riemannian metric $g$ on $M$ is compatible with $J$, that is $g(J X, Y)=g(X, J Y)$, for any $X, Y \in \mathfrak{X}(M)$, we call the pair $(g, J)$ metallic Riemannian structure and $(M, g, J)$ metallic Riemannian manifold.

It was shown in 12 that the powers of $J$ satisfy

$$
J^{n}=g_{n} J+q g_{n-1} I_{\mathfrak{X}(M)},
$$

where $\left\{g_{n}\right\}_{n \in \mathbb{N}^{*}}$ is the generalized secondary Fibonacci sequence defined by $g_{n+1}=$ $p g_{n}+q g_{n-1}, n \geq 1$, with $g_{0}=0, g_{1}=1$ and $p, q \in \mathbb{N}^{*}$.

Remark 1.1. i) The idea of introducing a metallic structure 12 as a solution of the equation $J^{2}-p J-q I_{\mathfrak{X}(M)}=0$ for $p, q \in \mathbb{N}^{*}$ was based on the fact that the positive root of the corresponding equation $x^{2}-p x-q=0$ is precisely a metallic number for $p, q \in \mathbb{N}^{*}$. By letting $p$ and $q$ be any natural number, we also include some other well-known structures; for instance, if $(p, q) \in\{(0,0),(0,1),(1,0)\}$, the solution of (1.1) would yield an almost tangent, an almost product, and a $J(2,1)$-structure, respectively.

ii) Concerning the inheritance of the metallic structure on submanifolds, Hreţcanu and Crasmareanu proved in 12 that a metallic structure on a metallic Riemannian 
manifold $M$ induces a metallic structure on every invariant submanifold of $M$ (i.e., on those submanifolds $N$ of $M$ for which $J\left(T_{x} N\right) \subset T_{x} N$, for any $\left.x \in N\right)$ and illustrate this on a product of spheres in an Euclidean space.

Fix now $J$ a metallic structure on $M$ and define the associated linear connections as follows.

Definition 1.2. $\nabla \in \mathcal{C}(M)$ is a $J$-connection if $J$ is covariant constant with respect to $\nabla$, namely $\nabla J=0$. Let $\mathcal{C}_{J}(M)$ be the set of these connections.

The concept of integrability is defined in the classical manner:

Definition 1.3. A metallic structure $J$ is called integrable if its Nijenhuis tensor field $N_{J}(X, Y):=[J X, J Y]-J[J X, Y]-J[X, J Y]+J^{2}[X, Y]$ vanishes.

Necessary and sufficient conditions for the integrability of a polynomial structure $J$ whose characteristic polynomial has only simple roots were given by Vanzura in [15], who proved that if there exists a symmetric linear $J$-connection $\nabla$, then the structure $J$ is integrable.

In order to find a measure of how far away a linear connection is from being in $\mathcal{C}_{J}(M)$ we introduce in the next section the notion of metallic conjugate connection. The present paper deals with the study of these connections and then it is the fifth in a series containing [4], 5], 3] and [6]. We remark that Bejan and Crasmareanu [1] studied the conjugate connections with respect to a quadratic endomorphism as a generalization of almost complex and almost product cases. An important tool in our work is provided by the pair (structural tensor, virtual tensor) defined for the almost product geometry in [5] and considered here in the last part of Section 1. After treating in Section 2 the duality between the metallic and the product conjugate connections, from an applied point of view we consider in Section 3 the $J$-invariant distributions.

\section{Metallic conjugate connection}

Let $\nabla$ be a linear connection on the metallic manifold $(M, J)$ and define the metallic conjugate connection of $\nabla$ by:

$$
\nabla^{(J)}:=\nabla+J \circ \nabla J
$$

It results that if $\nabla \in \mathcal{C}_{J}(M)$ then $\nabla^{(J)} \in \mathcal{C}_{J}(M)$. A direct computation gives us the expression of $\nabla^{(J)}$ :

$\nabla_{X}^{(J)} Y=\nabla_{X} Y+J\left(\nabla_{X} J Y-J\left(\nabla_{X} Y\right)\right)=(1-q) \nabla_{X} Y-p J\left(\nabla_{X} Y\right)+J\left(\nabla_{X} J Y\right)$.

Recall that given a linear connection $\nabla$, one defines its torsion as the $(1,2)$-tensor field

$$
T_{\nabla}(X, Y):=\nabla_{X} Y-\nabla_{Y} X-[X, Y]
$$

and its curvature as the $(1,3)$-tensor field

$$
R_{\nabla}(X, Y, Z):=\nabla_{X} \nabla_{Y} Z-\nabla_{Y} \nabla_{X} Z-\nabla_{[X, Y]} Z
$$

for any $X, Y, Z \in \mathfrak{X}(M)$. We call $\nabla$ a symmetric (respectively, flat) connection if $T_{\nabla}=0$ (respectively, $R_{\nabla}=0$ ). 
Some properties of the metallic conjugate connection are given in the next proposition.

Proposition 2.1. Let $J$ be a metallic structure on the smooth manifold $M, \nabla a$ linear connection on $M$ and $\nabla^{(J)}$ the metallic conjugate connection of $\nabla$. Then:

(1) $\nabla^{(J)} J=\left((1-2 q) I_{\mathfrak{X}(M)}-p J\right) \circ \nabla J$.

(2) $T_{\nabla^{(J)}}=T_{\nabla}+J\left(d^{\nabla} J\right)$, where $d^{\nabla}$ is the exterior covariant derivative induced by $\nabla$, namely $\left(d^{\nabla} J\right)(X, Y):=\left(\nabla_{X} J\right) Y-\left(\nabla_{Y} J\right) X$.

(3) $R_{\nabla^{(J)}}(X, Y, Z)=\left((1-q) I_{\mathfrak{X}(M)}-p J\right)\left(R_{\nabla}(X, Y, Z)\right)+J\left(R_{\nabla}(X, Y, J Z)\right)+$ $\left.(1-q)\left(\left(F^{\nabla} J\right)(X, Y ; J Z)+\left(J-p I_{\mathfrak{X}(M)}\right)\left(F^{\nabla} J\right)(X, Y ; Z)\right)\right)$, where $\left(F^{\nabla} J\right)(X, Y ; Z):=\left(\nabla_{X} J\right) \nabla_{Y} Z-\left(\nabla_{Y} J\right) \nabla_{X} Z$.

(4) If $(M, g, J)$ is a metallic Riemannian manifold, then:

$$
\begin{aligned}
\left(\nabla_{X}^{(J)} g\right)(Y, Z)= & (1-q)\left(\nabla_{X} g\right)(Y, Z)-p\left(\nabla_{X} g\right)(Y, J Z) \\
& +\left(\nabla_{X} g\right)(J Y, J Z)-p g\left(Y,\left(\nabla_{X} J\right) Z\right) .
\end{aligned}
$$

It results that if $\nabla$ is a g-metric connection belonging to $\mathcal{C}_{J}(M)$ then $\nabla^{(J)}$ is also a g-metric connection (belonging to $\mathcal{C}_{J}(M)$ ).

Proof. (1) Observe that

$$
J\left(\left(\nabla_{X} J\right) J Y\right)=J\left(\nabla_{X} J^{2} Y\right)-J^{2}\left(\nabla_{X} J Y\right)=-q\left(\nabla_{X} J\right) Y,
$$

so

$$
\begin{aligned}
\left(\nabla_{X}^{(J)} J\right) Y:= & \nabla_{X}^{(J)} J Y-J\left(\nabla_{X}^{(J)} Y\right) \\
:= & (1-q) \nabla_{X} J Y-p J\left(\nabla_{X} J Y\right)+J\left(\nabla_{X} J^{2} Y\right) \\
& -(1-q) J\left(\nabla_{X} Y\right)+p J^{2}\left(\nabla_{X} Y\right)-J^{2}\left(\nabla_{X} J Y\right) \\
= & (1-q)\left(\nabla_{X} J Y-J\left(\nabla_{X} Y\right)\right)-p J\left(\nabla_{X} J Y-J\left(\nabla_{X} Y\right)\right) \\
& +J\left(\nabla_{X} J^{2} Y-J\left(\nabla_{X} J Y\right)\right) \\
:= & (1-q)\left(\nabla_{X} J\right) Y-p J\left(\left(\nabla_{X} J\right) Y\right)+J\left(\left(\nabla_{X} J\right) J Y\right) \\
= & (1-q)\left(\nabla_{X} J\right) Y-p J\left(\left(\nabla_{X} J\right) Y\right)-q\left(\nabla_{X} J\right) Y
\end{aligned}
$$

which yields the claimed formula.

(2)

$$
\begin{aligned}
T_{\nabla^{(J)}}(X, Y):= & \nabla_{X}^{(J)} Y-\nabla_{Y}^{(J)} X-[X, Y] \\
= & (1-q)\left(\nabla_{X} Y-\nabla_{Y} X\right)-p J\left(\nabla_{X} Y-\nabla_{Y} X\right)+J\left(\nabla_{X} J Y-\nabla_{Y} J X\right) \\
& +T_{\nabla}(X, Y)-\left(\nabla_{X} Y-\nabla_{Y} X\right) \\
= & -J^{2}\left(\nabla_{X} Y-\nabla_{Y} X\right)+J\left(\nabla_{X} J Y-\nabla_{Y} J X\right)+T_{\nabla}(X, Y) \\
= & J\left(\left(\nabla_{X} J\right) Y-\left(\nabla_{Y} J\right) X\right)+T_{\nabla}(X, Y) \\
= & J\left(\left(d^{\nabla} J\right)(X, Y)\right)+T_{\nabla}(X, Y) .
\end{aligned}
$$


(3) Notice that

$$
\begin{aligned}
\nabla_{X}^{(J)} \nabla_{Y}^{(J)} Z= & (1-q)^{2} \nabla_{X} \nabla_{Y} Z-(1-q) p \nabla_{X} J\left(\nabla_{Y} Z\right)+(1-q) \nabla_{X} J\left(\nabla_{Y} J Z\right) \\
& -p J\left(\nabla_{X} \nabla_{Y} Z\right)+(1-q) J\left(\nabla_{X} J\left(\nabla_{Y} Z\right)\right)+q J\left(\nabla_{X} \nabla_{Y} J Z\right)
\end{aligned}
$$

and the expression of curvature becomes

$$
\begin{aligned}
R_{\nabla^{(J)}} & (X, Y, Z):=\nabla_{X}^{(J)} \nabla_{Y}^{(J)} Z-\nabla_{Y}^{(J)} \nabla_{X}^{(J)} Z-\nabla_{[X, Y]}^{(J)} Z \\
= & (1-q)^{2}\left(R_{\nabla}(X, Y, Z)+\nabla_{[X, Y]} Z\right)-(1-q) \nabla_{[X, Y]} Z \\
& -p J\left(R_{\nabla}(X, Y, Z)+\nabla_{[X, Y]} Z\right) \\
& +p J\left(\nabla_{[X, Y]} Z\right)+q J\left(R_{\nabla}(X, Y, J Z)+\nabla_{[X, Y]} J Z\right)-J\left(\nabla_{[X, Y]} J\right) \\
& -(1-q) p\left(\left(\nabla_{X} J\right) \nabla_{Y} Z-\left(\nabla_{Y} J\right) \nabla_{X} Z\right) \\
& -(1-q) p J\left(R_{\nabla}(X, Y, Z)+\nabla_{[X, Y]} Z\right) \\
& +(1-q)\left(\left(\nabla_{X} J\right) \nabla_{Y} J Z-\left(\nabla_{Y} J\right) \nabla_{X} J Z\right) \\
& +(1-q) J\left(R_{\nabla}(X, Y, J Z)+\nabla_{[X, Y]} J\right) \\
& +(1-q) J\left(\left(\nabla_{X} J\right) \nabla_{Y} Z-\left(\nabla_{Y} J\right) \nabla_{X} Z\right) \\
& +(1-q) J^{2}\left(R_{\nabla}(X, Y, Z)+\nabla_{[X, Y]} Z\right) \\
= & \left((1-q) I_{\mathfrak{X}(M)}-p J\right) R_{\nabla}(X, Y, Z)+J\left(R_{\nabla}(X, Y, J Z)\right) \\
& +(1-q)\left(\left(F^{\nabla} J\right)(X, Y ; J Z)+\left(J-p I_{\mathfrak{X}(M)}\right)\left(F^{\nabla} J\right)(X, Y ; Z)\right) .
\end{aligned}
$$

(4) Using that $g(J X, Y)=g(X, J Y)$ and $g(J X, J Y)=p g(X, J Y)+q g(X, Y)$ we obtain:

$$
\begin{aligned}
\left(\nabla_{X}^{(J)} g\right) & (Y, Z):=X(g(Y, Z))-g\left(\nabla_{X}^{(J)} Y, Z\right)-g\left(Y, \nabla_{X}^{(J)} Z\right) \\
= & X(g(Y, Z))-(1-q)\left(X(g(Y, Z))-\left(\nabla_{X} g\right)(Y, Z)\right) \\
& +\left(\nabla_{X} g\right)(J Y, J Z)-X(g(J Y, J Z)) \\
& +p\left(X(g(Y, J Z))-\left(\nabla_{X} g\right)(Y, J Z)-g\left(Y, \nabla_{X} J Z\right)+g\left(Y, J\left(\nabla_{X} Z\right)\right)\right) \\
= & (1-q)\left(\nabla_{X} g\right)(Y, Z)-p\left(\nabla_{X} g\right)(Y, J Z)+\left(\nabla_{X} g\right)(J Y, J Z) \\
& +q X(g(Y, Z))+p X(g(Y, J Z))-X(g(J Y, J Z))-p g\left(Y,\left(\nabla_{X} g\right) Z\right) \\
= & (1-q)\left(\nabla_{X} g\right)(Y, Z)-p\left(\nabla_{X} g\right)(Y, J Z)+\left(\nabla_{X} g\right)(J Y, J Z)-p g\left(Y,\left(\nabla_{X} g\right) Z\right) .
\end{aligned}
$$

Recall that a linear connection $\nabla$ is called quarter-symmetric connection [10] if its torsion is of the form $T_{\nabla}(X, Y)=\omega(X) T(Y)-T(X) \omega(Y)$, for any $X, Y \in$ $\mathfrak{X}(M)$, where $\omega$ is a 1 -form and $T$ is a $(1,1)$-tensor field.

A natural generalization of the case $\nabla \in \mathcal{C}_{J}(M)$ is given by:

Proposition 2.2. Let $\nabla$ be a symmetric linear connection. Assume that $\nabla J=$ $\eta \otimes J^{n}$, for $n \in \mathbb{N}$, where $\eta$ is a 1-form. Then $\nabla^{(J)}=\nabla+\eta \otimes J^{n+1}$ and it is a quarter-symmetric connection.

Proof. From 2.1 we obtain $\nabla^{(J)}=\nabla+\eta \otimes J^{n+1}$ and $T_{\nabla^{(J)}}=\eta \otimes J^{n+1}-J^{n+1} \otimes \eta$, which gives us the conclusion. 
Definition 2.3. A symmetric linear connection $\nabla$ is called special metallic connection if $d^{\nabla} J=0$.

Denote by $\mathcal{C}_{\mathfrak{M}}(M)$ the set of all special metallic connections on $M$. We remark that:

i) $\mathcal{C}_{\mathfrak{M}}(M)$ contains the symmetric $J$-connections;

ii) for $\nabla \in \mathcal{C}_{\mathfrak{M}}(M)$, its conjugate connection $\nabla^{(J)}$ is symmetric.

Proposition 2.4. If $\nabla$ is a special metallic connection, then:

1. $\nabla^{(J)}$ is special metallic, too.

2. The Nijenhuis tensor field of $J$ vanishes, i.e., $J$ is integrable.

Proof. 1. It is a consequence of Proposition 2.1

2. A straightforward computation gives:

$$
N_{J}(X, Y)=\left(d^{\nabla} J\right)(J X, Y)-\left(d^{\nabla} J\right)(J Y, X)-p\left(d^{\nabla} J\right)(X, Y)
$$

and the result follows from $d^{\nabla} J=0$.

Definition 2.5. We say that $(g, J, \nabla)$ is a special metallic structure if $(g, J)$ is a metallic Riemannian structure and $\nabla$ is a special metallic connection.

We remark that for a given pair $(\nabla, J)$, because of the duality between the metallic and the almost product structures (see Section 2), there always exists a Riemannian metric $g$ such that $(g, J, \nabla)$ is a special metallic structure.

Example 2.1. The following examples of special metallic structures on the 5-dimensional Euclidean space can be generalized to special metallic structures defined on Euclidean spaces of any odd dimension.

Let $\mathbb{R}^{5}=\mathbb{R}^{2} \times \mathbb{R}^{3}$ be the Euclidean space with the metric given by the Euclidean scalar product $g=\langle\cdot, \cdot\rangle$ and denote by $\left(x^{1}, x^{2}, y^{1}, y^{2}, y^{3}\right)$ the local coordinates in $\mathbb{R}^{5}$.

For any fixed $p, q \in \mathbb{N}^{*}$, define the $(1,1)$-tensor field $J_{0}: \mathfrak{X}\left(\mathbb{R}^{5}\right) \rightarrow \mathfrak{X}\left(\mathbb{R}^{5}\right)$ by

$$
J_{0}\left(\frac{\partial}{\partial x_{1}}, \frac{\partial}{\partial x_{2}}, \frac{\partial}{\partial y_{1}}, \frac{\partial}{\partial y_{2}}, \frac{\partial}{\partial y_{3}}\right):=\left(\sigma \frac{\partial}{\partial x_{1}}, \sigma \frac{\partial}{\partial x_{2}}, \bar{\sigma} \frac{\partial}{\partial y_{1}}, \bar{\sigma} \frac{\partial}{\partial y_{2}}, \bar{\sigma} \frac{\partial}{\partial y_{3}}\right),
$$

where $\sigma:=\sigma_{p, q}=\frac{p+\sqrt{p^{2}+4 q}}{2}$ is the $(p, q)$-metallic number and $\bar{\sigma}=\frac{p-\sqrt{p^{2}+4 q}}{2}=p-\sigma$.

We can check that $J_{0}$ is a metallic structure on $\mathbb{R}^{5}$ :

$$
\begin{aligned}
J_{0}^{2}\left(X^{1}, X^{2}, Y^{1}, Y^{2}, Y^{3}\right): & =\left(\sigma^{2} X^{1}, \sigma^{2} X^{2}, \bar{\sigma}^{2} Y^{1}, \bar{\sigma}^{2} Y^{2}, \bar{\sigma}^{2} Y^{3}\right) \\
& =p\left(\sigma X^{1}, \sigma X^{2}, \bar{\sigma} Y^{1}, \bar{\sigma} Y^{2}, \bar{\sigma} Y^{3}\right)+q\left(X^{1}, X^{2}, Y^{1}, Y^{2}, Y^{3}\right),
\end{aligned}
$$

for any $\left(X^{1}, X^{2}, Y^{1}, Y^{2}, Y^{3}\right) \in \mathfrak{X}\left(\mathbb{R}^{5}\right)$. Thus, $J_{0}^{2}=p J_{0}+q I_{\mathfrak{X}\left(\mathbb{R}^{5}\right)}$.

Moreover, the metric is $J_{0}$-compatible:

$$
g\left(J_{0} Z, Z^{\prime}\right)=\sigma\left(X^{1} X^{\prime 1}+X^{2} X^{\prime 2}\right)+\bar{\sigma}\left(Y^{1} Y^{\prime 1}+Y^{2} Y^{\prime 2}+Y^{3} Y^{\prime 3}\right)=g\left(Z, J_{0} Z^{\prime}\right),
$$

for any $Z:=\left(X^{1}, X^{2}, Y^{1}, Y^{2}, Y^{3}\right), Z^{\prime}=\left(X^{\prime 1}, X^{\prime 2}, Y^{\prime 1}, Y^{\prime 2}, Y^{\prime 3}\right) \in \mathfrak{X}\left(\mathbb{R}^{5}\right)$. Thus, $\left(\mathbb{R}^{5}, g, J_{0}\right)$ is a metallic Riemannian manifold. 
In a similar manner we can verify that the $(1,1)$-tensor fields $J_{1}, J_{2}, J_{3}, J_{4}$ : $\mathfrak{X}\left(\mathbb{R}^{5}\right) \rightarrow \mathfrak{X}\left(\mathbb{R}^{5}\right)$ defined by

$$
\begin{aligned}
& J_{1}\left(\frac{\partial}{\partial x_{1}}, \frac{\partial}{\partial x_{2}}, \frac{\partial}{\partial y_{1}}, \frac{\partial}{\partial y_{2}}, \frac{\partial}{\partial y_{3}}\right):=\left(\sigma \frac{\partial}{\partial x_{1}}, \sigma \frac{\partial}{\partial x_{2}}, \sigma \frac{\partial}{\partial y_{1}}, \sigma \frac{\partial}{\partial y_{2}}, \bar{\sigma} \frac{\partial}{\partial y_{3}}\right), \\
& J_{2}\left(\frac{\partial}{\partial x_{1}}, \frac{\partial}{\partial x_{2}}, \frac{\partial}{\partial y_{1}}, \frac{\partial}{\partial y_{2}}, \frac{\partial}{\partial y_{3}}\right):=\left(\bar{\sigma} \frac{\partial}{\partial x_{1}}, \sigma \frac{\partial}{\partial x_{2}}, \bar{\sigma} \frac{\partial}{\partial y_{1}}, \bar{\sigma} \frac{\partial}{\partial y_{2}}, \bar{\sigma} \frac{\partial}{\partial y_{3}}\right), \\
& J_{3}\left(\frac{\partial}{\partial x_{1}}, \frac{\partial}{\partial x_{2}}, \frac{\partial}{\partial y_{1}}, \frac{\partial}{\partial y_{2}}, \frac{\partial}{\partial y_{3}}\right):=\left(\bar{\sigma} \frac{\partial}{\partial x_{1}}, \bar{\sigma} \frac{\partial}{\partial x_{2}}, \sigma \frac{\partial}{\partial y_{1}}, \sigma \frac{\partial}{\partial y_{2}}, \sigma \frac{\partial}{\partial y_{3}}\right), \\
& J_{4}\left(\frac{\partial}{\partial x_{1}}, \frac{\partial}{\partial x_{2}}, \frac{\partial}{\partial y_{1}}, \frac{\partial}{\partial y_{2}}, \frac{\partial}{\partial y_{3}}\right):=\left(\bar{\sigma} \frac{\partial}{\partial x_{1}}, \sigma \frac{\partial}{\partial x_{2}}, \sigma \frac{\partial}{\partial y_{1}}, \sigma \frac{\partial}{\partial y_{2}}, \bar{\sigma} \frac{\partial}{\partial y_{3}}\right)
\end{aligned}
$$

are metallic structures on $\mathbb{R}^{5}$ and $\left(\mathbb{R}^{5}, g, J_{i}\right), i \in\{1, \ldots, 4\}$, are metallic Riemannian manifolds.

Since the Levi-Civita $\nabla^{g}$ is trivial, $\left(g, J_{i}, \nabla^{g}\right), i \in\{0, \ldots, 4\}$, are special metallic structures on $\mathbb{R}^{5}$.

Example 2.2. In a similar way, one can construct special metallic structures on the Euclidean spaces of even dimension.

Let $\mathbb{R}^{4}=\mathbb{R}^{2} \times \mathbb{R}^{2}$ be the Euclidean space with the metric given by the Euclidean scalar product $g=\langle\cdot, \cdot\rangle$ and denote by $\left(x^{1}, x^{2}, y^{1}, y^{2}\right)$ the local coordinates in $\mathbb{R}^{4}$. In a similar manner as in Example 2.1, we can check that for given $p, q \in \mathbb{N}^{*}$, the $(1,1)$-tensor fields $J_{0}^{\prime}: \mathfrak{X}\left(\mathbb{R}^{4}\right) \rightarrow \mathfrak{X}\left(\mathbb{R}^{4}\right)$ defined by

$$
\begin{aligned}
& J_{0}^{\prime}\left(\frac{\partial}{\partial x_{1}}, \frac{\partial}{\partial x_{2}}, \frac{\partial}{\partial y_{1}}, \frac{\partial}{\partial y_{2}}\right):=\left(\sigma \frac{\partial}{\partial x_{1}}, \bar{\sigma} \frac{\partial}{\partial x_{2}}, \bar{\sigma} \frac{\partial}{\partial y_{1}}, \bar{\sigma} \frac{\partial}{\partial y_{2}}\right), \\
& J_{1}^{\prime}\left(\frac{\partial}{\partial x_{1}}, \frac{\partial}{\partial x_{2}}, \frac{\partial}{\partial y_{1}}, \frac{\partial}{\partial y_{2}}\right):=\left(\bar{\sigma} \frac{\partial}{\partial x_{1}}, \bar{\sigma} \frac{\partial}{\partial x_{2}}, \sigma \frac{\partial}{\partial y_{1}}, \sigma \frac{\partial}{\partial y_{2}}\right), \\
& J_{2}^{\prime}\left(\frac{\partial}{\partial x_{1}}, \frac{\partial}{\partial x_{2}}, \frac{\partial}{\partial y_{1}}, \frac{\partial}{\partial y_{2}}\right):=\left(\bar{\sigma} \frac{\partial}{\partial x_{1}}, \sigma \frac{\partial}{\partial x_{2}}, \bar{\sigma} \frac{\partial}{\partial y_{1}}, \bar{\sigma} \frac{\partial}{\partial y_{2}}\right), \\
& J_{3}^{\prime}\left(\frac{\partial}{\partial x_{1}}, \frac{\partial}{\partial x_{2}}, \frac{\partial}{\partial y_{1}}, \frac{\partial}{\partial y_{2}}\right):=\left(\sigma \frac{\partial}{\partial x_{1}}, \sigma \frac{\partial}{\partial x_{2}}, \bar{\sigma} \frac{\partial}{\partial y_{1}}, \bar{\sigma} \frac{\partial}{\partial y_{2}}\right), \\
& J_{4}^{\prime}\left(\frac{\partial}{\partial x_{1}}, \frac{\partial}{\partial x_{2}}, \frac{\partial}{\partial y_{1}}, \frac{\partial}{\partial y_{2}}\right):=\left(\sigma \frac{\partial}{\partial x_{1}}, \bar{\sigma} \frac{\partial}{\partial x_{2}}, \sigma \frac{\partial}{\partial y_{1}}, \bar{\sigma} \frac{\partial}{\partial y_{2}}\right)
\end{aligned}
$$

are metallic structures on $\mathbb{R}^{4}$ and $\left(\mathbb{R}^{4}, g, J_{i}^{\prime}\right), i \in\{0, \ldots, 4\}$, are metallic Riemannian manifolds. Thus $\left(g, J_{i}^{\prime}, \nabla^{g}\right), i \in\{0, \ldots, 4\}$, are special metallic structures on $\mathbb{R}^{4}$.

The last subject of this section treats two tensor fields associated to a metallic structure. The paper [5] introduces the structural and virtual tensor fields of an almost product structure. Turning into our framework, let us consider for a pair $(\nabla, J)$ the following tensor fields of $(1,2)$-type:

1) the structural tensor field

$$
C_{\nabla}^{J}(X, Y):=\frac{1}{2}\left[\left(\nabla_{J X} J\right) Y+\left(\nabla_{X} J\right) J Y\right]
$$


2) the virtual tensor field

$$
B_{\nabla}^{J}(X, Y):=\frac{1}{2}\left[\left(\nabla_{J X} J\right) Y-\left(\nabla_{X} J\right) J Y\right]
$$

It results that

$$
\begin{aligned}
C_{\nabla^{(J)}}^{J}(X, Y):= & \frac{1}{2}\left[\left(\nabla_{J X}^{(J)} J\right) Y+\left(\nabla_{X}^{(J)} J\right) J Y\right] \\
= & \frac{1}{2}\left[(1-2 q)\left(\nabla_{J X} J\right) Y-p J\left(\left(\nabla_{J X} J\right) Y\right)\right. \\
& \left.+(1-2 q)\left(\nabla_{X} J\right) J Y-p J\left(\left(\nabla_{X} J\right) J Y\right)\right] \\
:= & (1-2 q) C_{\nabla}^{J}(X, Y)-p J\left(C_{\nabla}^{J}(X, Y)\right)
\end{aligned}
$$

and

$$
\begin{aligned}
B_{\nabla^{(J)}}^{J}(X, Y):= & \frac{1}{2}\left[\left(\nabla_{J X}^{(J)} J\right) Y-\left(\nabla_{X}^{(J)} J\right) J Y\right] \\
= & \frac{1}{2}\left[(1-2 q)\left(\nabla_{J X} J\right) Y-p J\left(\left(\nabla_{J X} J\right) Y\right)\right. \\
& \left.-(1-2 q)\left(\nabla_{X} J\right) J Y+p J\left(\left(\nabla_{X} J\right) J Y\right)\right] \\
:= & (1-2 q) B_{\nabla}^{J}(X, Y)-p J\left(B_{\nabla}^{J}(X, Y)\right),
\end{aligned}
$$

hence

$$
C_{\nabla^{(J)}}^{J}=\left((1-2 q) I_{\mathfrak{X}(M)}-p J\right) \circ C_{\nabla}^{J}, \quad B_{\nabla^{(J)}}^{J}=\left((1-2 q) I_{\mathfrak{X}(M)}-p J\right) \circ B_{\nabla}^{J} .
$$

Also,

$$
\left(\nabla_{J^{2} X} J\right) J Y=p^{2} \nabla_{J X} J Y+p q \nabla_{J X} Y-p J\left(\nabla_{J X} J Y\right)+q\left(\nabla_{X} J\right) J Y
$$

and

$$
\left(\nabla_{J X} J\right) J^{2} Y=p^{2} \nabla_{J X} J Y+p q \nabla_{J X} Y-p J\left(\nabla_{J X} J Y\right)+q\left(\nabla_{J X} J\right) Y,
$$

which implies

$$
\begin{aligned}
C_{\nabla}^{J}(J X, J Y) & :=\frac{1}{2}\left[\left(\nabla_{J^{2} X} J\right) J Y+\left(\nabla_{J X} J\right) J^{2} Y\right] \\
& =p^{2} \nabla_{J X} J Y+p q \nabla_{J X} Y-p J\left(\nabla_{J X} J Y\right)+q C_{\nabla}^{J}(X, Y) \\
& =p\left(\nabla_{J X} J^{2} Y-J\left(\nabla_{J X} J Y\right)\right)+q C_{\nabla}^{J}(X, Y) \\
& :=p\left(\nabla_{J X} J\right) J Y+q C_{\nabla}^{J}(X, Y)
\end{aligned}
$$

and

$$
\begin{aligned}
B_{\nabla}^{J}(J X, J Y) & :=\frac{1}{2}\left[\left(\nabla_{J^{2} X} J\right) J Y-\left(\nabla_{J X} J\right) J^{2} Y\right]=\frac{1}{2} q\left(\left(\nabla_{X} J\right) J Y-\left(\nabla_{J X} J\right) Y\right) \\
& :=-q B_{\nabla}^{J}(X, Y),
\end{aligned}
$$

hence

$$
C_{\nabla}^{J}(J X, J Y)=q C_{\nabla}^{J}(X, Y)+p\left(\nabla_{J X} J\right) J Y, \quad B_{\nabla}^{J}(J X, J Y)=-q B_{\nabla}^{J}(X, Y) .
$$

The importance of these tensor fields for our study is underlined by the following relation:

$$
\nabla^{(J)}=\nabla+p \nabla J-C_{\nabla}^{J}+B_{\nabla}^{J}
$$


Example 2.3. If the linear connection $\nabla$ satisfies $\nabla J=\eta \otimes J^{n}$, for $n \in \mathbb{N}$, where $\eta$ is a 1 -form, then the structural and the virtual tensor fields have the expressions

$$
\begin{gathered}
C_{\nabla}^{J}=\frac{1}{2}\left[(\eta \circ J) \otimes J^{n}+\eta \otimes J^{n+1}\right], \quad B_{\nabla}^{J}=\frac{1}{2}\left[(\eta \circ J) \otimes J^{n}-\eta \otimes J^{n+1}\right], \\
C_{\nabla^{(J)}}^{J}=\frac{1}{2}\left[(\eta \circ J) \otimes\left(\left((1-2 q) I_{\mathfrak{X}(M)}-p J\right) \circ J^{n}\right)+\eta \otimes\left(\left((1-2 q) I_{\mathfrak{X}(M)}-p J\right) \circ J^{n+1}\right)\right], \\
B_{\nabla^{(J)}}^{J}=\frac{1}{2}\left[(\eta \circ J) \otimes\left(\left((1-2 q) I_{\mathfrak{X}(M)}-p J\right) \circ J^{n}\right)-\eta \otimes\left(\left((1-2 q) I_{\mathfrak{X}(M)}-p J\right) \circ J^{n+1}\right)\right] .
\end{gathered}
$$

Example 2.4. Concerning the behavior of $\nabla^{(.)}$for families of metallic structures, remark that if $J_{1}$ and $J_{2}$ are two metallic structures with $J_{1}^{2}=p J_{1}+q_{1} I_{\mathfrak{X}(M)}$ and $J_{2}^{2}=p J_{2}+q_{2} I_{\mathfrak{X}(M)}$, then $J:=J_{1}+J_{2}$ is a metallic structure with $J^{2}=p J+q I_{\mathfrak{X}(M)}$ if and only if $J_{1} J_{2}+J_{2} J_{1}=\left(q-q_{1}-q_{2}\right) I_{\mathfrak{X}(M)}$; in this case:

$$
\begin{gathered}
\nabla_{X}^{\left(J_{1}+J_{2}\right)} Y=\nabla_{X}^{\left(J_{1}\right)} Y+\nabla_{X}^{\left(J_{2}\right)} Y-\left(1+q-q_{1}-q_{2}\right) \nabla_{X} Y+J_{1}\left(\nabla_{X} J_{2} Y\right)+J_{2}\left(\nabla_{X} J_{1} Y\right), \\
C_{\nabla}^{J_{1}+J_{2}}-B_{\nabla}^{J_{1}+J_{2}}=\left(C_{\nabla}^{J_{1}}+C_{\nabla}^{J_{2}}\right)-\left(B_{\nabla}^{J_{1}}+B_{\nabla}^{J_{2}}\right)-J_{1} \circ \nabla J_{2}-J_{2} \circ \nabla J_{1} .
\end{gathered}
$$

\section{The duality of metallic and Product CONJugate COnNections}

In [12] Hreţcanu and Crasmareanu proved that any metallic structure $J$ induces two almost product structures:

$$
E_{ \pm}= \pm\left(\frac{2}{2 \sigma_{p, q}-p} J-\frac{p}{2 \sigma_{p, q}-p} I_{\mathfrak{X}(M)}\right),
$$

and for any given pair $(p, q) \in \mathbb{N}^{*} \times \mathbb{N}^{*}$, any almost product structure $E$ determines two metallic structures:

$$
J_{ \pm}= \pm\left(\frac{2 \sigma_{p, q}-p}{2}\right) E+\frac{p}{2} I_{\mathfrak{X}(M)} .
$$

Then $\nabla E_{ \pm}= \pm \frac{2}{2 \sigma_{p, q}-p} \nabla J$ and $\nabla J_{ \pm}= \pm \frac{2 \sigma_{p, q}-p}{2} \nabla E$ respectively. Hence, $\nabla$ is a $J$-connection if and only if $\nabla$ is an $E$-connection.

We are interested in finding the relation between the conjugate connections associated to them.

Proposition 3.1. i) If $J$ is a metallic structure on $M$ and $E_{ \pm}$are the almost product structures given by (3.1), then

$$
\left(2 \sigma_{p, q}-p\right)^{2} \nabla^{\left(E_{ \pm}\right)}-4 \nabla^{(J)}-\left(\left(2 \sigma_{p, q}-p\right)^{2}-4\right) \nabla=-2 p \nabla J .
$$

ii) If $E$ is an almost product structure on $M$ and $J_{ \pm}$are the metallic structures given by $(3.2)$, then

$$
4 \nabla^{\left(J_{ \pm}\right)}-\left(2 \sigma_{p, q}-p\right)^{2} \nabla^{(E)}-\left(\left(2 \sigma_{p, q}-p\right)^{2}-4\right) \nabla= \pm p\left(2 \sigma_{p, q}-p\right) \nabla E .
$$

iii) In each of the previous cases, it holds $\left(\nabla^{(J)}\right)^{(E)}=\left(\nabla^{(E)}\right)^{(J)}$. 
Proof. i) Replacing $E_{ \pm}$from 3.1 in the expression of $\nabla^{\left(E_{ \pm}\right)}([5])$ :

$$
\nabla_{X}^{\left(E_{ \pm}\right)} Y=E_{ \pm}\left(\nabla_{X} E_{ \pm} Y\right)
$$

we get

$$
\begin{aligned}
\nabla_{X}^{\left(E_{ \pm}\right)} Y= & \frac{4}{\left(2 \sigma_{p, q}-p\right)^{2}} J\left(\nabla_{X} J Y\right)-\frac{2 p}{\left(2 \sigma_{p, q}-p\right)^{2}} J\left(\nabla_{X} Y\right) \\
& -\frac{2 p}{\left(2 \sigma_{p, q}-p\right)^{2}} \nabla_{X} J Y+\frac{p^{2}}{\left(2 \sigma_{p, q}-p\right)^{2}} \nabla_{X} Y
\end{aligned}
$$

and replacing $J\left(\nabla_{X} J Y\right)=\nabla_{X}^{(J)} Y-(1-q) \nabla_{X} Y+p J\left(\nabla_{X} Y\right)$ we obtain

$$
\begin{aligned}
\nabla_{X}^{\left(E_{ \pm}\right)} Y= & \frac{4}{\left(2 \sigma_{p, q}-p\right)^{2}} \nabla_{X}^{(J)} Y-\frac{4(1-q)-p^{2}}{\left(2 \sigma_{p, q}-p\right)^{2}} \nabla_{X} Y \\
& -\frac{2 p}{\left(2 \sigma_{p, q}-p\right)^{2}}\left(\nabla_{X} J Y-J\left(\nabla_{X} Y\right)\right) \\
= & \frac{4}{\left(2 \sigma_{p, q}-p\right)^{2}} \nabla_{X}^{(J)} Y+\frac{\left(2 \sigma_{p, q}-p\right)^{2}-4}{\left(2 \sigma_{p, q}-p\right)^{2}} \nabla_{X} Y \\
& -\frac{2 p}{\left(2 \sigma_{p, q}-p\right)^{2}}\left(\nabla_{X} J\right) Y .
\end{aligned}
$$

ii) Replacing $J_{ \pm}$from 3.2 in

$$
\nabla_{X}^{\left(J_{ \pm}\right)} Y=(1-q) \nabla_{X} Y-p J_{ \pm}\left(\nabla_{X} Y\right)+J_{ \pm}\left(\nabla_{X} J_{ \pm} Y\right)
$$

we get

$$
\begin{aligned}
\nabla_{X}^{\left(J_{ \pm}\right)} Y= & \left(1-q-\frac{p^{2}}{4}\right) \nabla_{X} Y \pm \frac{p\left(2 \sigma_{p, q}-p\right)}{4}\left(\nabla_{X} E Y-E\left(\nabla_{X} Y\right)\right) \\
& +\frac{\left(2 \sigma_{p, q}-p\right)^{2}}{4} E\left(\nabla_{X} E Y\right)
\end{aligned}
$$

and replacing $E\left(\nabla_{X} E Y\right)=\nabla_{X}^{(E)} Y$ we obtain

$$
\nabla_{X}^{\left(J_{ \pm}\right)} Y=\frac{\left(2 \sigma_{p, q}-p\right)^{2}}{4} \nabla_{X}^{(E)} Y-\frac{\left(2 \sigma_{p, q}-p\right)^{2}-4}{4} \nabla_{X} Y \pm \frac{p\left(2 \sigma_{p, q}-p\right)}{4}\left(\nabla_{X} E\right) Y
$$

iii) From a direct computation we obtain

$$
\left(\nabla^{(J)}\right)_{X}^{\left(E_{ \pm}\right)} Y=(1-q) E_{ \pm}\left(\nabla_{X} E_{ \pm} Y\right)-p\left(E_{ \pm} \circ J\right)\left(\nabla_{X} E_{ \pm} Y\right)+\left(E_{ \pm} \circ J\right)\left(\nabla_{X}\left(J \circ E_{ \pm}\right) Y\right)
$$

and respectively

$\left(\nabla^{\left(E_{ \pm}\right)}\right)_{X}^{(J)} Y=(1-q) E_{ \pm}\left(\nabla_{X} E_{ \pm} Y\right)-p\left(J \circ E_{ \pm}\right)\left(\nabla_{X} E_{ \pm} Y\right)+\left(J \circ E_{ \pm}\right)\left(\nabla_{X}\left(E_{ \pm} \circ J\right) Y\right)$, and taking into account that $J \circ E_{ \pm}=E_{ \pm} \circ J$, we get $\left(\nabla^{(J)}\right)^{\left(E_{ \pm}\right)}=\left(\nabla^{\left(E_{ \pm}\right)}\right)^{(J)}$.

Also,

$$
\left(\nabla^{(E)}\right)_{X}^{\left(J_{ \pm}\right)} Y=(1-q) E\left(\nabla_{X} E Y\right)-p\left(J_{ \pm} \circ E\right)\left(\nabla_{X} E Y\right)+\left(J_{ \pm} \circ E\right)\left(\nabla_{X}\left(E \circ J_{ \pm}\right) Y\right)
$$

and respectively

$\left(\nabla^{\left(J_{ \pm}\right)}\right)_{X}^{(E)} Y=(1-q) E\left(\nabla_{X} E Y\right)-p\left(E \circ J_{ \pm}\right)\left(\nabla_{X} E Y\right)+\left(E \circ J_{ \pm}\right)\left(\nabla_{X}\left(J_{ \pm} \circ E\right) Y\right)$, 
and taking into account that $E \circ J_{ \pm}=J_{ \pm} \circ E$, we get $\left(\nabla^{(E)}\right)^{\left(J_{ \pm}\right)}=\left(\nabla^{\left(J_{ \pm}\right)}\right)^{(E)}$.

We showed in [5] that for an almost product structure $E$, the structural and virtual tensor fields satisfy

$$
\nabla^{(E)}=\nabla-C_{\nabla}^{E}+B_{\nabla}^{E}
$$

From 2.4 and 3.3 we obtain:

Corollary 3.2. i) If $J$ is a metallic structure on $M$ and $E_{ \pm}$are the almost product structures given by (3.1), then

$$
\left(2 \sigma_{p, q}-p\right)^{2}\left(C_{\nabla}^{E_{ \pm}}-B_{\nabla}^{E_{ \pm}}\right)=4\left(C_{\nabla}^{J}-B_{\nabla}^{J}\right)-2 p \nabla J .
$$

ii) If $E$ is an almost product structure on $M$ and $J_{ \pm}$are the metallic structures given by 3.2 , then

$4\left(C_{\nabla}^{J_{ \pm}}-B_{\nabla}^{J_{ \pm}}\right)=\left(2 \sigma_{p, q}-p\right)^{2}\left(C_{\nabla}^{E}-B_{\nabla}^{E}\right) \pm p\left(2 \sigma_{p, q}-p\right) \nabla E-2\left(\left(2 \sigma_{p, q}-p\right)^{2}-4\right) \nabla$.

Proof. Replacing the expressions

$$
\nabla^{(J)}=\nabla-C_{\nabla}^{J}+B_{\nabla}^{J}+p \nabla J
$$

and

$$
\nabla^{(E)}=\nabla-C_{\nabla}^{E}+B_{\nabla}^{E}
$$

in the two relations stated in Proposition 3.1. we get the required relations between the structural and the virtual tensors.

\section{INVARIANT DISTRIBUTIONS}

Let $J$ be a metallic structure on $M$ and $\mathcal{D}_{l}$ and $\mathcal{D}_{m}$ be the complementary orthogonal distributions corresponding to the projection operators ([12]):

$$
l=\frac{\sigma_{p, q}}{2 \sigma_{p, q}-p} \cdot I-\frac{1}{2 \sigma_{p, q}-p} \cdot J, \quad m=\frac{\sigma_{p, q}-p}{2 \sigma_{p, q}-p} \cdot I+\frac{1}{2 \sigma_{p, q}-p} \cdot J .
$$

Notice that the operators $l$ and $m$ verify the following equalities:

$$
\begin{gathered}
l+m=I, \quad l^{2}=l, \quad m^{2}=m, \quad l m=m l=0, \\
J l=l J=\left(p-\sigma_{p, q}\right) l, \quad J m=m J=\sigma_{p, q} m .
\end{gathered}
$$

Definition 4.1. Let $\mathcal{D} \subset T M$ be a distribution considered as a vector subbundle of $T M$. We say that:

i) the distribution $\mathcal{D}$ is $J$-invariant if $X \in \Gamma(\mathcal{D})$ implies $J X \in \Gamma(\mathcal{D})$;

ii) ([7]) the linear connection $\nabla$ restricts to $\mathcal{D}$ if $Y \in \Gamma(\mathcal{D})$ implies $\nabla_{X} Y \in \Gamma(\mathcal{D})$, for any $X \in \Gamma(T M)$.

If $\nabla$ restricts to $\mathcal{D}$, then $\nabla$ may be considered as a connection in the vector bundle $\mathcal{D}$. From this fact, in [2] a connection which restricts to $\mathcal{D}$ is called adapted to $\mathcal{D}$.

Proposition 4.2. If the linear connection $\nabla$ restricts to $\mathcal{D}_{l}$ (respectively, $\mathcal{D}_{m}$ ), then $\nabla^{(J)}$ also restricts to $\mathcal{D}_{l}$ (respectively, $\mathcal{D}_{m}$ ). 
Proof. Fix $Y \in \Gamma\left(\mathcal{D}_{l}\right)$. Then $J Y \in \Gamma\left(\mathcal{D}_{l}\right)$ and for any $X \in \Gamma(T M)$, we have $\nabla_{X} Y \in \Gamma\left(\mathcal{D}_{l}\right)$. It follows that $J\left(\nabla_{X} Y\right), J\left(\nabla_{X} J Y\right) \in \Gamma\left(\mathcal{D}_{l}\right)$, so $\nabla_{X}^{(J)} Y=(1-$ q) $\nabla_{X} Y-p J\left(\nabla_{X} Y\right)+J\left(\nabla_{X} J Y\right) \in \Gamma\left(\mathcal{D}_{l}\right)$. Analogously for $\mathcal{D}_{m}$.

A more general notion is given by geodesic invariance [7]. The distribution $\mathcal{D}$ is $\nabla$-geodesically invariant if for every geodesic $\gamma:[a, b] \rightarrow M$ of $\nabla$ with $\dot{\gamma}(a) \in \mathcal{D}_{\gamma(a)}$ it follows that $\dot{\gamma}(t) \in \mathcal{D}_{\gamma(t)}$, for any $t \in[a, b]$. A necessary and sufficient condition for a distribution $\mathcal{D}$ to be $\nabla$-geodesically invariant is given in [7]: for any $X$ and $Y \in \Gamma(\mathcal{D})$, the symmetric product $\langle X: Y\rangle:=\nabla_{X} Y+\nabla_{Y} X$ to belong to $\Gamma(\mathcal{D})$ or equivalently, for any $X \in \Gamma(\mathcal{D})$ to have $\nabla_{X} X \in \Gamma(\mathcal{D})$.

The following result is a direct consequence of definitions:

Proposition 4.3. If the linear connection $\nabla$ restricts to $\mathcal{D}_{l}$ (respectively, $\mathcal{D}_{m}$ ), then $\mathcal{D}_{l}$ (respectively, $\mathcal{D}_{m}$ ) is geodesically invariant for $\nabla^{(J)}$.

We remark that $E:=l-m$ is an almost product structure and both $\mathcal{D}_{l}$ and $\mathcal{D}_{m}$ are $E$-invariant. The product conjugate connection of $\nabla$ is

$$
\nabla_{X}^{(E)} Y=l\left(\nabla_{X} l Y\right)-l\left(\nabla_{X} m Y\right)-m\left(\nabla_{X} l Y\right)+m\left(\nabla_{X} m Y\right)
$$

and then we have:

Proposition 4.4. If $\nabla^{(E)}$ is symmetric, then $E$ is integrable, which means that $\mathcal{D}_{l}$ and $\mathcal{D}_{m}$ are involutive distributions.

Proof. From 4.1 we get

$$
l[X, Y]+m[X, Y]=\nabla_{X}^{(E)} Y-\nabla_{Y}^{(E)} X=l\left(\nabla_{X} l Y-\nabla_{Y} l X\right)+m\left(\nabla_{X} l Y-\nabla_{Y} l X\right)
$$

and then

$$
l[X, Y]=\nabla_{X} l Y-\nabla_{Y} l X, \quad m[X, Y]=\nabla_{X} m Y-\nabla_{Y} m X .
$$

With $X \rightarrow m X$ and $Y \rightarrow m Y$ in the first relation above it follows that $l[m X, m Y]=$ 0 and the change $X \rightarrow l X$ and $Y \rightarrow m Y$ in the second relation yields $m[l X, l Y]=$ 0 .

We have:

1) $\nabla$ restricts to $\mathcal{D}_{l}$ means $m\left(\nabla_{X} l Y\right)=0$ and $l\left(\nabla_{X} l Y\right)=\nabla_{X} l Y$,

2) $\nabla$ restricts to $\mathcal{D}_{m}$ means $l\left(\nabla_{X} m Y\right)=0$ and $m\left(\nabla_{X} m Y\right)=\nabla_{X} m Y$.

A straightforward computation gives that $\nabla^{(E)}$ from 4.1 restricts to $\mathcal{D}_{l}$ and $\mathcal{D}_{m}$. Moreover, if $\nabla$ restricts to both $\mathcal{D}_{l}$ and $\mathcal{D}_{m}$, then

$$
\nabla_{X}^{(E)} Y=\nabla_{X} l Y+\nabla_{X} m Y=\nabla_{X} Y
$$

and so $\nabla \in \mathcal{C}_{E}(M)$. Let us remark that the above connection 4.2 is exactly the Schouten connection of the pair $(l, m)$ [8]:

$$
\nabla_{X} Y=l\left(\nabla_{X} l Y\right)+m\left(\nabla_{X} m Y\right) .
$$

We shall express the structural and the virtual tensor fields of the almost product structure $E$ in terms of the projectors $l, m$ as follows: 
Proposition 4.5. The structural and virtual tensor fields of $E:=l-m$ are:

$$
\left\{\begin{array}{l}
C_{\nabla}^{l-m}(X, Y)=2\left[l\left(\nabla_{m X} m Y\right)+m\left(\nabla_{l X} l Y\right)\right] \\
B_{\nabla}^{l-m}(X, Y)=-2\left[l\left(\nabla_{l X} m Y\right)+m\left(\nabla_{m X} l Y\right)\right] .
\end{array}\right.
$$

Proof. From [5] we know that

$$
C_{\nabla}^{E}(E X, E Y)=C_{\nabla}^{E}(X, Y), B_{\nabla}^{E}(E X, E Y)=-B_{\nabla}^{E}(X, Y)
$$

and we get

$$
\left\{\begin{aligned}
C_{\nabla}^{l-m}(l X, m Y) & =-C_{\nabla}^{l-m}(m X, l Y) \\
B_{\nabla}^{l-m}(l X, l Y) & =-B_{\nabla}^{l-m}(m X, m Y) .
\end{aligned}\right.
$$

By making $X \rightarrow m X$ in the first relation and $X \rightarrow l X$ in the second one, it results that

$$
\left\{\begin{array}{l}
C_{\nabla}^{l-m}(l X, m Y)=0=C_{\nabla}^{l-m}(m X, l Y) \\
B_{\nabla}^{l-m}(l X, m Y)=0=B_{\nabla}^{l-m}(m X, m Y)
\end{array}\right.
$$

and then

$$
\left\{\begin{array}{l}
C_{\nabla}^{l-m}(X, Y)=C_{\nabla}^{l-m}(l X, l Y)+C_{\nabla}^{l-m}(m X, m Y) \\
B_{\nabla}^{l-m}(X, Y)=B_{\nabla}^{l-m}(l X, l Y)+B_{\nabla}^{l-m}(m X, m Y) .
\end{array}\right.
$$

But

$$
\left\{\begin{aligned}
C_{\nabla}^{l-m}(l X, l Y) & =2 m\left(\nabla_{l X} l Y\right) \\
C_{\nabla}^{l-m}(m X, m Y) & =2 l\left(\nabla_{m X} m Y\right)
\end{aligned}\right.
$$

and

$$
\left\{\begin{array}{l}
B_{\nabla}^{l-m}(l X, m Y)=-2 l\left(\nabla_{l X} m Y\right) \\
B_{\nabla}^{l-m}(m X, l Y)=-2 m\left(\nabla_{m X} l Y\right)
\end{array}\right.
$$

and then we have the conclusion.

Remark 4.1. If $T$ and $A$ are the fundamental tensor fields of O'Neill-Gray,

$$
\left\{\begin{array}{l}
T(X, Y)=l\left(\nabla_{m X} m Y\right)+m\left(\nabla_{m X} l Y\right) \\
A(X, Y)=m\left(\nabla_{l X} l Y\right)+l\left(\nabla_{l X} m Y\right)
\end{array}\right.
$$

a comparison of the last two equations yields

$$
\left\{\begin{array}{l}
C_{\nabla}^{l-m}(X, Y)=2[T(X, m Y)+A(X, l Y)] \\
B_{\nabla}^{l-m}(X, Y)=-2[T(X, l Y)+A(X, m Y)]
\end{array}\right.
$$

a fact which justifies the second name of $T$ and $A$ as invariants of the considered orthogonal decomposition of $T M$ [8].

\section{ACKNOWLEDGEMENT}

The authors wish to express thanks to the referee for the valuable suggestions and remarks that definitely improved the paper. 


\section{REFERENCES}

[1] C. L. Bejan and M. Crasmareanu, Conjugate connections with respect to a quadratic endomorphism and duality, Filomat 30 (2016), no. 9, 2367-2374. MR 3583413

[2] A. Bejancu and H. R. Farran, Foliations and geometric structures, Mathematics and Its Applications 580, Springer, Dordrecht, 2006. MR 2190039

[3] A. M. Blaga, The geometry of golden conjugate connections, Sarajevo J. Math. 10(23) (2014), no. 2, 237-245. MR 3280661.

[4] A. M. Blaga and M. Crasmareanu, The geometry of complex conjugate connections, Hacet. J. Math. Stat. 41 (2012), no. 1, 119-126. MR 2976917.

[5] A. M. Blaga and M. Crasmareanu, The geometry of product conjugate connections, An. Ştiint. Univ. Al. I. Cuza Iaşi. Mat. (N.S.) 59 (2013), no. 1, 73-84. MR 3098381

[6] A. M. Blaga and M. Crasmareanu, The geometry of tangent conjugate connections, Hacet. J. Math. Stat. 44 (2015), no. 4, 767-774. MR 3444107.

[7] F. Bullo and A. D. Lewis, Geometric control of mechanical systems. Modeling, analysis, and design for simple mechanical control systems, Texts in Applied Mathematics, 49, SpringerVerlag, New York, 2005. MR 2099139

[8] M. Falcitelli, S. Ianuş and A. M. Pastore, Riemannian submersions and related topics, World Scientific, River Edge, NJ, 2004. MR 2110043

[9] S. Falcon and A. Plaza, On the 3-dimensional k-Fibonacci spirals, Chaos Solitons Fractals 38 (2008), no. 4, 993-1003. MR 2435599

[10] S. Gołąb, On semi-symmetric and quarter-symmetric linear connections, Tensor (N.S.) 29 (1975), no. 3, 249-254. MR 0383275

[11] S. I. Goldberg and K. Yano, Polynomial structures on manifolds, Kōdai Math. Sem. Rep. 22 (1970), 199-218. MR 0267478.

[12] C.-E. Hreţcanu and M. Crasmareanu, Metallic structures on Riemannian manifolds, Rev. Un. Mat. Argentina 54 (2013), no. 2, 15-27. MR 3263648

[13] P. Singh, The so-called Fibonacci numbers in ancient and medieval India, Historia Math. 12 (1985), no. 3, 229-244. MR 0803579

[14] V. W. de Spinadel, The metallic means and design, Nexus II: Architecture and Mathematics (Mantua, 1998), 143-157. Collana “Gli Studi”, 5, Edizioni dell'Erba, Fucecchio, 1998. MR 1999340

[15] J. Vanžura, Integrability conditions for polynomial structures, Kōdai Math. Sem. Rep. 27 (1976), no. 1-2, 42-50. MR 0400106.

Adara M. Blaga ${ }^{凶}$

Department of Mathematics and Computer Sciences

West University from Timişoara

Bdl. V. Pârvan nr. 4, 300223 Timişoara, România

adarablaga@yahoo.com

Cristina-Elena Hreţcanu

Ştefan cel Mare University from Suceava

University Str., nr. 13, 720229 Suceava, România

criselenab@yahoo.com

Received: February 17, 2017

Accepted: September 15, 2017 